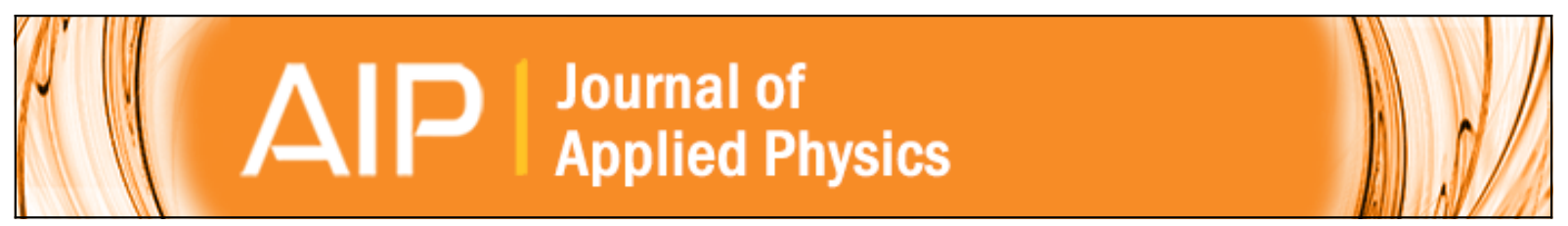

\title{
Towards an on-chip platform for the controlled application of forces via magnetic
} particles: A novel device for mechanobiology

M. Monticelli, E. Albisetti, D. Petti, D. V. Conca, M. Falcone, P. P. Sharma, and R. Bertacco

Citation: Journal of Applied Physics 117, 17B317 (2015); doi: 10.1063/1.4917191

View online: http://dx.doi.org/10.1063/1.4917191

View Table of Contents: http://scitation.aip.org/content/aip/journal/jap/117/17?ver=pdfcov

Published by the AIP Publishing

\section{Articles you may be interested in}

Magnetic approaches to study collective three-dimensional cell mechanics in long-term cultures (invited)

J. Appl. Phys. 115, 172616 (2014); 10.1063/1.4870918

Biofunctionalized magnetic nanoparticles for in vitro labeling and in vivo locating specific biomolecules Appl. Phys. Lett. 92, 142504 (2008); 10.1063/1.2907486

On-chip separation of magnetic particles with different magnetophoretic mobilities

J. Appl. Phys. 101, 024913 (2007); 10.1063/1.2423137

Magnetic cell manipulation utilizing magnetic particles and diamagnetic collagen fibers

J. Appl. Phys. 99, $08 R 906$ (2006); 10.1063/1.2175957

Superconducting quantum interference device-based magnetic nanoparticle relaxation measurement as a novel tool for the binding specific detection of biological binding reactions (abstract)

J. Appl. Phys. 81, 4317 (1997); 10.1063/1.364754

Frustrated by

old technology?

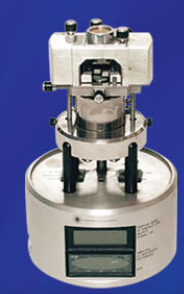

Is your AFM dead

and can't be repaired?

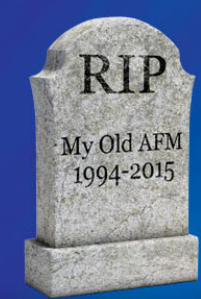

Sick of bad customer support?

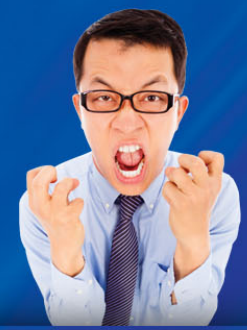

It is time to upgrade your AFM Minimum $\$ 20,000$ trade-in discount for purchases before August 31st

Asylum Research is today's technology leader in AFM

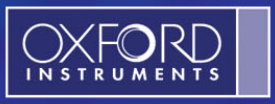




\title{
Towards an on-chip platform for the controlled application of forces via magnetic particles: A novel device for mechanobiology
}

\author{
M. Monticelli, E. Albisetti, ${ }^{a}$ D. Petti, D. V. Conca, M. Falcone, P. P. Sharma, and R. Bertacco \\ LNESS, Dipartimento di Fisica, Politecnico di Milano, Via Anzani 42, 22100 Como, Italy
}

(Presented 4 November 2014; received 23 September 2014; accepted 3 December 2014; published online 13 April 2015)

\begin{abstract}
In-vitro tests and analyses are of fundamental importance for investigating biological mechanisms in cells and bio-molecules. The controlled application of forces to activate specific bio-pathways and investigate their effects, mimicking the role of the cellular environment, is becoming a prominent approach in this field. In this work, we present a non-invasive magnetic on-chip platform which allows for the manipulation of magnetic particles, through micrometric magnetic conduits of Permalloy patterned on-chip. We show, from simulations and experiments, that this technology permits to exert a finely controlled force on magnetic beads along the chip surface. This force can be tuned from few to hundreds pN by applying a variable external magnetic field. ( 2015 AIP Publishing LLC .
\end{abstract}

[http://dx.doi.org/10.1063/1.4917191]

In recent years, mechanobiology has attracted a growing interest in the scientific community as it offers a new and unconventional approach to investigate the physiological and pathological behavior of cells and bio-entities. This emergent and multidisciplinary field paves the way to a deeper comprehension of specific molecular mechanisms that are actually under investigation and can be crucial for diagnostics and treatment of many diseases (e.g., cancer). Mechanobiology is based on the application of mechanical stimuli on target cells or biomolecules, in order to mimic in different conditions the role of extracellular matrix (ECM) in organs and tissues, highlighting specific relevant biopathways like genes expression or cellular migration.

In this field, many technologies have been developed for this purpose. A relevant example is the use of functional materials, ${ }^{1,2}$ like polydimethylsiloxane (PDMS) (Ref. 3) or gels, ${ }^{4}$ properly micro- and nanostructured to obtain in-vivo-like stimuli mimicking those arising from the ECM. These tools are intensively used in different mechanobiology studies as they can be easily fabricated and because they allow high parallelization in tests and analyses. However, in the most general cases, cells are exposed to spatially homogenous, constant, and predefined mechanical stimuli over time. An alternative is represented by micromechanical stretching devices, microfluidic, or microelectromechanical systems (MEMS), ${ }^{5,6}$ whose typical feature size matches the micrometric dimension of the mammalian cell. Nevertheless, they do not permit to localize and precisely tune the intensity of mechanical forces applied on the cells.

Another strategy is offered by patch-clamp ${ }^{7}$ and atomic force microscopy ${ }^{8}$ based technologies which exploit micrometric and nanometric tips to exert controlled forces. They allow a localized mechanical stimulation, but they are invasive, as they can easily damage cells and biomolecules and do not easily allow the parallelization of the experiment in statistical studies.

In this context, micromanipulators allowing the manipulation of micro- and nanoparticles to test cells and bio-

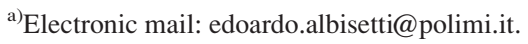

entities have been developed. One of the most widespread methods in biology is based on optical tweezers, ${ }^{9-11}$ which allows a fine confinement and regulation of the applied forces. However, this method can photo-damage the biological samples due to the extremely focalized light in lasers.

For this reason, magnetic manipulators have recently attracted a great interest because they are completely noninvasive for cells and biomolecules. Gunnarsson et al. ${ }^{12}$ first proposed to combine the action of an external magnetic field with ferromagnetic elements properly patterned on-chip, whose magnetization creates a confined stray field employed to attract and manipulate magnetic particles with a micrometric resolution. In 2009, an innovative magnetic handling technology, called "Domain Wall Tweezers" (DWT), ${ }^{13}$ has been proposed and patented ${ }^{14}$ by some of the authors. It is based on magnetic DWs displacement in ferromagnetic conduits. In such ferromagnetic conduits, magnetic domain walls act as attractive poles for magnetic particles, which can be trapped and displaced by applying external magnetic fields. This allows manipulation of magnetic micro and nanoparticles with resolution down to $100 \mathrm{~nm}$ (Refs. 15 and 16) and thus the development of a platform for mechanical actuation at the micro- and nano-scale.

Here, we present an on-chip device, based on the magnetic manipulation technology described in Refs. 13, 16, and 17 , which can be used to trap and manipulate superparamagnetic micro- and nano-particles in order to apply finely calibrated and localized stimuli. We demonstrate trough simulations and experiments that devices with micrometric ferromagnetic structures can exert a force along the chip surface on $1 \mu \mathrm{m}$ size bead, precisely tunable from few to several hundreds of $\mathrm{pN}$.

Simulations to estimate the force exerted by these magnetic tweezers have been performed by OOMMF (Object Oriented Micro Magnetic Framework). ${ }^{18}$ The micromagnetic configuration of the microstructures and the magnetic stray field exerted by the conduits has been calculated using the following parameters for $\mathrm{Ni}_{80} \mathrm{Fe}_{20}$ : saturation magnetization 
$M_{s}=680 \times 10^{3} \mathrm{~A} / \mathrm{m}$, exchange stiffness constant $A=1.3$ $\times 10^{-11} \mathrm{~J} / \mathrm{m}$, damping coefficient $\alpha=0.01$, no magnetocrystalline anisotropy has been considered. A $25 \mathrm{~nm} \times$ $25 \mathrm{~nm} \times 10 \mathrm{~nm}$ unit cell has been used for simulating the micromagnetic configuration of the zig-zag shaped and curved conduits. The dimension of the unit cell constitutes a reasonable compromise between the exchange length of $5.2 \mathrm{~nm}$ and sufficiently short computational time.

The magnetic force has been calculated from the micromagnetic configuration according to the following equation:

$$
\mathbf{F}_{\text {mag }}=-\mu_{0} \nabla \int_{V_{\text {bead }}}(\mathbf{M} \cdot \mathbf{H}) d V,
$$

where $\boldsymbol{M}$ is the magnetization of the superparamagnetic bead $(\boldsymbol{M}=\chi \boldsymbol{H})$ and $\boldsymbol{H}$ is the total field $\left(\boldsymbol{H}=\boldsymbol{H}_{\boldsymbol{c}}+\boldsymbol{H}_{\boldsymbol{e x t}}\right.$, calculated through OOMMF, taking into account the stray field generated by the ferromagnetic conduit $\left(\boldsymbol{H}_{\boldsymbol{c}}\right)$ and the external magnetic field $\left.\left(\boldsymbol{H}_{\text {ext }}\right)\right)$.

The bead is modeled as a single magnetic dipole placed in the geometrical center of the particle. In this way, the force can be written as follows:

$$
\mathbf{F}_{\text {mag }}=-\mu_{0} \chi \nabla \mathbf{H}^{2} V,
$$

where $V$ is the bead volume.

The device is presented in Figure 1. Magnetic conduits for beads trapping and manipulation are made of Permalloy $\left(\mathrm{Ni}_{80} \mathrm{Fe}_{20}\right)$. Two different geometries have been tested: rings and zig-zag shaped nanostructures. Both present a thickness
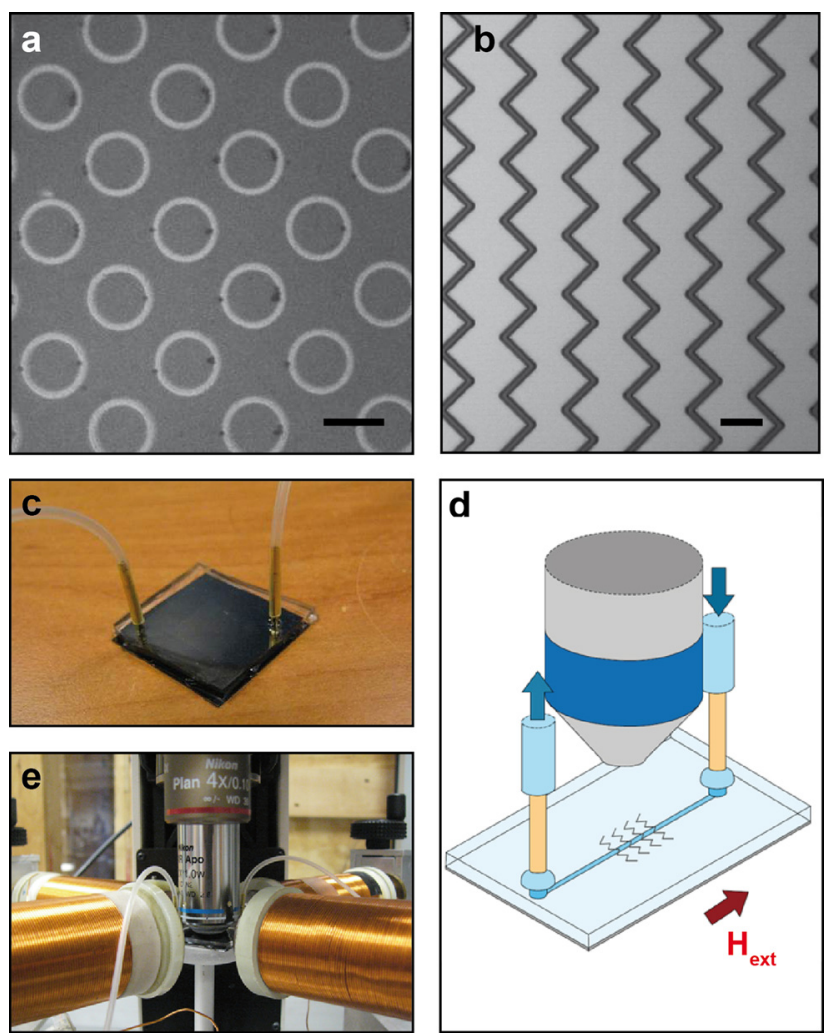

FIG. 1. Microscope images of rings (a) and zig-zag shaped (b) ferromagnetic Permalloy conduits. (c) Image of the microfluidic cell sealed on top of the patterned chip. (d) Sketch of the experimental setup. (e) Image of the measurement setup for force analysis. Scale bars: $20 \mu \mathrm{m}$ (a); $15 \mu \mathrm{m}$ (b). of $40 \mathrm{~nm}$; zig-zags are $2 \mu \mathrm{m}$ wide and have a segment length of $15 \mu \mathrm{m}$; rings are $2 \mu \mathrm{m}$ wide and have a $10 \mu \mathrm{m}$ radius (see Figs. 1(a) and 1(b)). The magnetic nanostructures are grown on the top of a Si substrate and fabricated by optical lithography, e-beam evaporation, and lift off-procedure. The nanostructures have been uniformly covered by a capping layer of $\mathrm{Si}_{3} \mathrm{~N}_{4}$ (40 nm thick) and $\mathrm{SiO}_{2}(10 \mathrm{~nm}$ thick) in order to isolate and prevent damaging due to the liquid where beads are suspended. A PDMS microfluidic channel, fabricated by soft-lithography from a SU-8 mold, is sealed on top of the chip. The channel, bonded on the chip by means of an $\mathrm{O}_{2}$ plasma treatment, is $2 \mathrm{~cm}$ long, $450 \mu \mathrm{m}$ wide, and $32.5 \mu \mathrm{m}$ thick. The microfluidic channel contains the liquid where magnetic particles are suspended (see Fig. 1(c)).

In order to test the device, commercial MyOne ${ }^{\circledR}$-dynabeads (Invitrogen, cross-linked polystyrene matrix, magnetization of saturation $M_{s}=40 \times 10^{6} \mathrm{~A} / \mathrm{m}$ and magnetic susceptibility $\chi=1.43), 1 \mu \mathrm{m}$ diameter, superparamagnetic particles functionalized with $\mathrm{COOH}$ have been used.

They have been diluted in a $\mathrm{H}_{2} \mathrm{O}$ environment $(\eta=0.89$ $\left.\times 10^{-3} \mathrm{~Pa}^{*} \mathrm{~s}\right)$ to reach a final concentration of $1 \mu \mathrm{g} / \mathrm{ml}$. The external field has been provided by a four-pole electromagnet able to provide a uniform field up to 1000 Oe in the sample plane. The experiment has been monitored under an optical microscope equipped with a $60 \times$ immersion objective and an EMCCD camera (see Figs. 1(d) and 1(e)). In order to control the flow of the liquid inside the channel and its velocity, a syringe pump system has been employed.

During the experiments, first, magnetic particles are conveyed in the microfluidic cell and trapped by the magnetic stray field arising from the Py structures when an external field is applied. Then, the fluid velocity is set by the syringe pump. When the dragging force exerted on the beads by the liquid is higher than the $x$-component of the magnetic force, beads are flushed away from the top of the structures. The $x$ component of the magnetic force has been estimated by measuring the minimum hydrodynamic force exerted by the liquid on the particles causing their detachment from the conduit. This dragging force, in laminar regime as the one that occurs in a microfluidic channel, is described by the Stokes equation

$$
\mathbf{F}_{\text {drag }}=6 \lambda \pi \eta r K \mathbf{v},
$$

where $v$ is the average fluid velocity in the channel, $\eta$ the medium viscosity, and $r$ the bead radius. $K$ is a parameter which depends on the velocity profile of the fluid. $\lambda$ is a correction coefficient which takes into account the vicinity of the wall. ${ }^{19}$ In fact, for a given fluid velocity, a higher dragging force is experimented by beads trapped in proximity to the surface compared to the case in which they are positioned in the middle of the channel. According to the equation in Ref. $19, \lambda$ is approximately equal to 3 when beads are in contact with the chip surface.

Figure 2 shows the micromagnetic configurations for ring (panel (a)) and zig-zag (panel (b)) structures when a 200 Oe external magnetic field is applied along the $x$-direction.

The equilibrium magnetization is determined by the interplay between the effect of the external field, which tends to align the magnetization along the $x$-direction, and the shape 


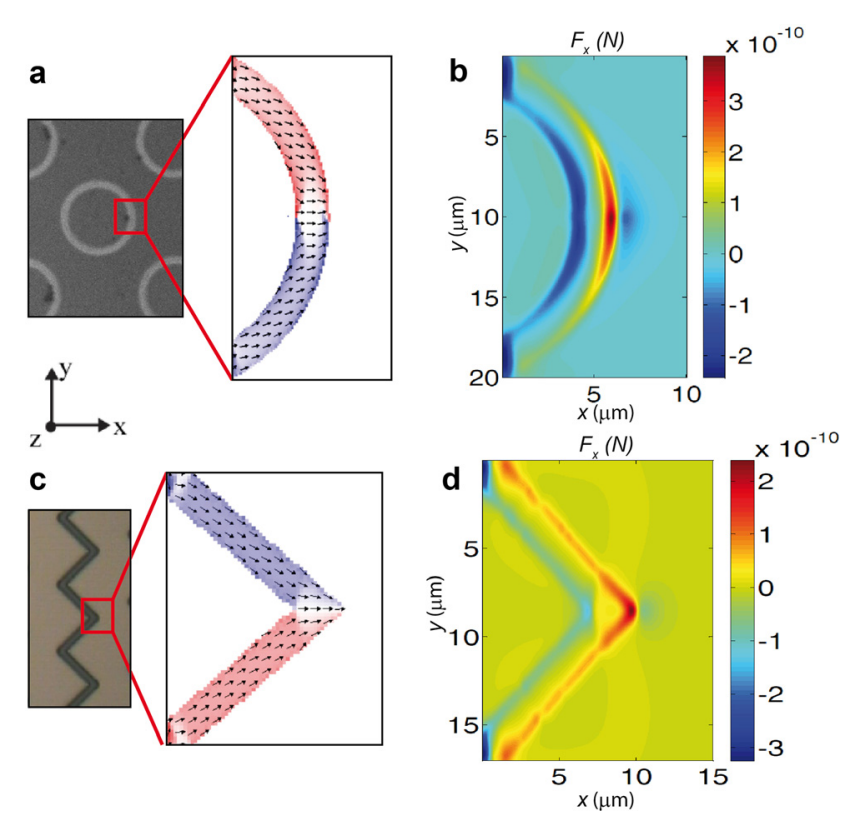

FIG. 2. Micromagnetic configuration calculated by OOMMF for rings (a) and zig-zag (c) shaped conduits. A 200 Oe external magnetic field is applied in the $x$-direction. The arrows represent the magnetization direction, while the pixels in red-white-blue scale the magnetization along y-axis. (b) and (d) Magnetic force along the $x$-direction acting on a $1 \mu \mathrm{m}$ magnetic bead at a distance of $60 \mathrm{~nm}$ from the conduits, for the ring and zig-zag structures, respectively.

anisotropy, which tends to orient the magnetization along the edges of the structure. As a result, the magnetization is locally directed in the sample plane, perpendicularly to the conduit direction (white pixels in panels (a) and (c)), producing an intense magnetic stray field gradient, which attracts superparamagnetic particles suspended in the liquid. In previous works, ${ }^{13,16}$ it has been demonstrated that the application of a sequence of magnetic fields pulses (for zig-zags) or a continuous rotational field (for rings) allows for trapping, release, and manipulation of magnetic particles along the entire conduit.

The $x$-component of the magnetic force exerted by magnetic tweezers on $1 \mu \mathrm{m}$ beads, i.e., the component of the magnetic force which counteracts the dragging force, has been simulated for the two geometries. When a 200 Oe external field $\left(\boldsymbol{H}_{\boldsymbol{e x t}}\right)$ is applied, the maximum value of the trapping force in the direction of the field ( $x$-direction) is $245 \mathrm{pN}$ for zig-zag shaped microstructures and $363 \mathrm{pN}$ for magnetic rings. Contour plots in Figures 2(b) and 2(d) show the force intensity experimented by the bead in the $x$-direction, considering a distance of $60 \mathrm{~nm}$ between the bead surface and the magnetic conduits. Compared with previous works where nanometric structures have been tested, ${ }^{13,16}$ the force profile is less spatially confined due to the micrometric width of the Py conduits. However, in our micrometric structures, the maximum force obtained is much higher. This fact is relevant because different biological mechanisms can be activated only by means of forces in the order of hundreds of $\mathrm{pN}$. ${ }^{1}$

In agreement with Eq. (2), $\boldsymbol{H}_{\text {ext }}$ affects the force experimented by beads. For magnetic rings, the force has been calculated as function of $\boldsymbol{H}_{\text {ext }}$ (from $50 \mathrm{Oe}$ to $300 \mathrm{Oe}$ ) and it is presented in Figure 3(c). In this case, the force ranges from 100 to $453 \mathrm{pN}$.

Therefore, it is shown that the magnetic force on the beads can be finely tuned by applying an external magnetic field.

To validate the estimated magnetic force, we measured the minimum flow rate of the liquid (precisely set by the syringe pump) which caused detachment of all the beads.

The test, described in this section, has been carried out varying the liquid flow rate from 40 to $250 \mu \mathrm{l} / \mathrm{min}$. In these conditions, a threshold flow rate for which all the beads are flushed away can be measured with a precision of $5 \mu \mathrm{l} / \mathrm{min}$. One has to note that isolate detaching events occur also for lower value of the flux due to Brownian motion and fabrication defects, but these are related to the statistical nature of the phenomenon and are not statistical meaningful. When $\boldsymbol{H}_{\text {ext }}$ of $200 \mathrm{Oe}$ is applied, the detaching flux is $175 \mu \mathrm{l} / \mathrm{min}$ for rings and $95 \mu \mathrm{l} / \mathrm{min}$ for zig-zags.

From the flow rate, the average velocity of the fluid in the channel is $v=\phi / A$ ( $\phi$ is the flux, $A$ the section area of the
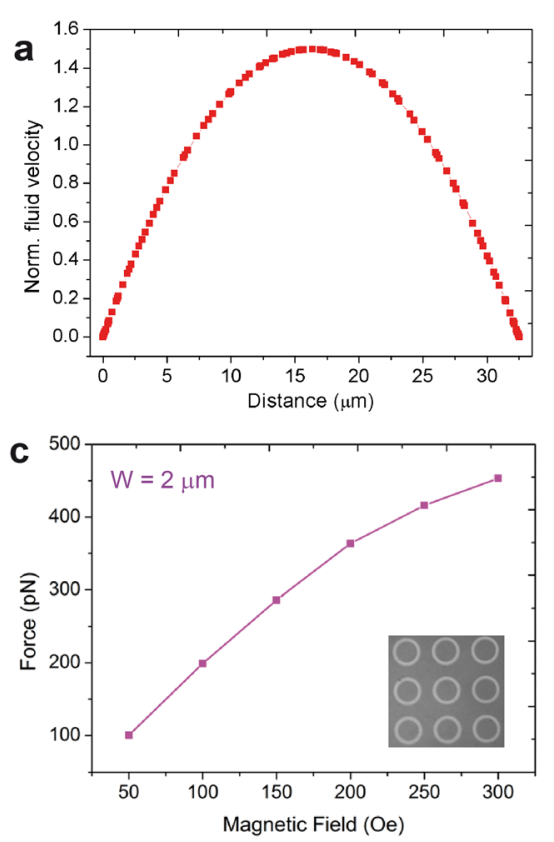

b
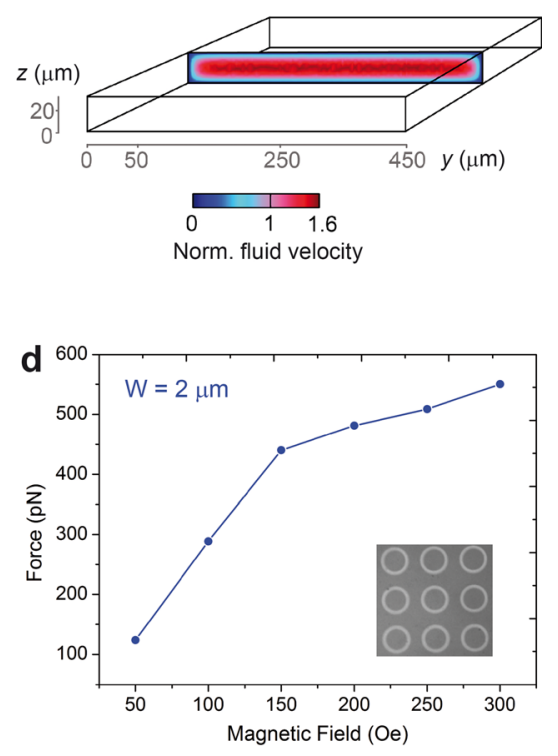

FIG. 3. (a) Velocity of the fluid in the microfluidic channel, as function of the distance from the chip surface, normalized to the average fluid velocity. (b) Sketch of the velocity profile within the channel. Magnetic force along the $x$-direction in ring shaped structures, simulated by OOMMF (c) and evaluated from experiments (d) as a function of the external magnetic field. 
microfluidic channel) and the force experimented by beads can be calculated using the Stokes equation (Eq. (3)), considering the fluid velocity profiles inside the channel reported in Figures 3(a) and 3(b) for a $35 \mu \mathrm{m}$ high channel. It is worth noting that the liquid velocity, in the laminar regime, presents a maximum in the middle of the channel and decreases to zero close to the channel boundaries (see the normalized velocity as a function of the distance from the chip surface, panel (a), and the velocity profile in the channel section, panel (b)). Therefore, considering only the velocity profile, the hydrodynamic force experimented by the bead trapped on the chip surface is lower compared to the hydrodynamic force exerted in the middle of the channel. However, this effect is partially compensated by the wall effect, which is included in the force expression through the $\lambda$ coefficient. ${ }^{19}$ The value of the force has been calculated from the velocity profile, integrating over the bead, placed at $10 \mathrm{~nm}$ from the channel bottom. According to the velocity profile, it is also possible to write the dragging force directly from the Stokes equation with $K=0.085$; this parameter does not depend on the average velocity of the fluid in the microfluidic cell, but only on the channel geometry.

For the zig-zag shaped structure, the detaching force along the $x$-direction $\left(\boldsymbol{F}_{\boldsymbol{x}}\right)$ is $265 \pm 10 \mathrm{pN}$, while for rings $\boldsymbol{F}_{\boldsymbol{x}}$ is $481 \pm 10 \mathrm{pN}$. Compared to zig-zag conduits, the ringshaped structure exerts a higher and less spatially confined force, due to the larger portion of magnetization directed perpendicular to the conduit (see Figure 2).

In order to evaluate the effect of the magnetic field on the forces exerted by the ring-shaped microstructures, the experiment has been repeated in the same microfluidic channel for different values of the external magnetic field. Magnetic field values ranging from 50 Oe to 300 Oe correspond to a detaching flow rate ranging from 45 to $200 \mu \mathrm{l} / \mathrm{min}$ and therefore to forces from $124 \pm 10 \mathrm{pN}$ to $550 \pm 10 \mathrm{pN}$, as illustrated in Figure 3(d). The uncertainty in the force estimation is ascribed to the error in the flow rate measurements, to some non-idealities in the channel geometry due to fabrication imperfections, and to approximations in the model employed for evaluating $\lambda .{ }^{19} \mathrm{It}$ is worth to notice that the values of the forces found experimentally are in good agreement with the simulations. The small discrepancy between the experimental and theoretical results can be explained by considering that the particles are modeled as single magnetic dipole and that we neglected both their Brownian motion and their non-specific interactions with the surface.

In order to understand the impact of the Brownian motion on the bead trapping and manipulation, we employed the model of Refs. 20 and 21, considering a Boltzmann distribution, including a thermal contribution to the total potential energy of the bead. However, the magnetic potential well generated by the conduits is $10^{4}$ times higher than the thermal energy associated with the bead, resulting in a mean displacement due to Brownian motion lower than $50 \mathrm{~nm}$.

Apart from the aforementioned discrepancies, the experimental results illustrated in Fig. 3(d) confirm the simulation finding, i.e., that it is possible to finely tune the magnetic force varying the value of $\boldsymbol{H}_{\text {ext }}$. Moreover, even higher forces can be generated increasing the bead volume, its magnetic momentum, or the Py thickness in the magnetic conduits. Furthermore, the capping layer could be made thinner, in order to reduce the distance between the beads and the magnetic layer, compatibly with the need of protecting the magnetic layer from the solution. In this way, it could be possible to realize a compact, non-invasive platform for the controlled application of forces in the $\mathrm{nN}$ range.

In summary, we have presented a magnetic on-chip technology based on ferromagnetic (Py) conduits patterned on chip, which allows for the trapping and manipulation of magnetic beads, exerting finely controlled and localized forces that can be tuned under the action of an external magnetic field in the $\mathrm{pN}$ range. The values of the forces parallel to the chip surface, acting on a $1 \mu \mathrm{m}$ superparamagnetic bead, have been extrapolated from experiments and from simulations, for two different nanostructures geometries (zig-zag shaped and rings). While zig-zag conduits exert more confined forces, ring structures generate forces of higher intensities up to $200 \mathrm{pN}$.

As an example of application, in-vitro experiments can be envisioned where single magnetic micro- and nanoparticles are brought in contact with specific cells, and subsequently are manipulated for applying a localized controlled stimulus to the cellular membrane.

This platform holds the potential to realize a new noninvasive tool for the application of spatially controlled, tunable forces up to the $\mathrm{nN}$ range for different in-vitro biological studies in the field of mechanobiology.

This work was funded by the Fondazione Centro Europeo di Nanomedicina through the project "Forces, mechanisms and pathways involved in the ATR-mediated control of nuclear plasticity in response to mechanical stress" (EP002), and by Fondazione Cariplo via the project UMANA (20130735).

${ }^{1}$ Y. Shao and J. Fu, Adv. Mater. 26, 1494-1533 (2014).

${ }^{2}$ S. Ghassemi et al., PNAS 109(14), 5328-5333 (2012).

${ }^{3}$ C. S. Simmons et al., J. Micromech. Microeng. 21, 054016 (2011).

${ }^{4}$ W. J. Polacheck et al., Lab Chip 13, 2252 (2013).

${ }^{5}$ D.-H. Kim et al., Annu. Rev. Biomed. Eng. 11, 203 (2009).

${ }^{6}$ J. Rajagopalan and M. T. Saif, J. Micromech. Microeng. 21, 54002 (2011).

${ }^{7}$ P. Hinterdorfer and Y. F. Dufrene, Nat. Methods 3, 347 (2006).

${ }^{8}$ A. Kishino and T. Yanagida, Nature 334, 74 (1988).

${ }^{9}$ A. Ashkin, Phys. Rev. Lett. 24, 156 (1970).

${ }^{10} \mathrm{~A}$. Ashkin, "Forces of a single-beam gradient laser trap on a dielectric sphere in the ray optics regime," in Methods in Cell Biology (Academic Press, 1998), Vol. 55.

${ }^{11}$ S. M. Block et al., Nature 348, 348 (1990).

${ }^{12} \mathrm{~K}$. Gunnarsson et al., Adv. Mater. 17, 1730 (2005).

${ }^{13}$ M. Donolato et al., Adv. Mater. 22, 2706 (2010).

${ }^{14} \mathrm{R}$. Bertacco et al., "Manipulation of magnetic particles in magnetic conduits for the propagation of domain walls," U.S. patent No. 13148649 (16 February 2012).

${ }^{15}$ A. Torti et al., Appl. Phys. Lett. 101, 142405 (2012).

${ }^{16}$ M. Donolato et al., Lab Chip 11, 2976 (2011).

${ }^{17}$ P. Vavassori et al., Appl. Phys. Lett. 93, 203502 (2008).

${ }^{18}$ D. M. J. Donahue, “1999 OOMMF User's Guide, Version 1.0,” Interagency Report NISTIR No. 6376.3, 2004.

${ }^{19}$ P. Holmqvist et al., J. Chem. Phys. 126, 044707 (2007).

${ }^{20}$ K. V. Ommeringa et al., Appl. Phys. Lett. 89, 142511 (2006).

${ }^{21}$ L. E. Helseth et al., Langmuir 20, 6556-6559 (2004). 